\title{
Towards an Accurate Identification of Pyloric Neuron Activity with VSDi
}

\author{
Filipa dos Santos and Peter Andras and KP Lam
}

School of Computing and Mathematics, University of Keele, STAFFS ST5 5BG, UK

\{f.dos.santos, p.andras, k.p.lam\}@keele.ac.uk

\begin{abstract}
Voltage-sensitive dye imaging (VSDi) which enables simultaneous optical recording of many neurons in the pyloric circuit of the stomatogastric ganglion is an important technique to supplement electrophysiological recordings. However, utilising the technique to identify pyloric neurons directly is a computationally exacting task that requires the development of sophisticated signal processing procedures to analyse the tri-phasic pyloric patterns generated by these neurons. This paper presents our work towards commissioning such procedures. The results achieved to date are most encouraging.
\end{abstract}

Keywords: Duty Cycle, Tri-phasic Pyloric Neural Network, Voltage-Sensitive Dye Imaging, Singular Spectrum Analysis, Dynamic phase detection.

\section{$1 \quad$ Introduction}

In recent years, an optical recording technique based on voltage-sensitive dye imaging (VSDi) has become a practical means of simultaneously capturing the activities of multiple neurons in living tissues, providing a valuable tool for studying neural circuit connectivity and neuromodulation at the single cell level. This is exemplified by recent studies of the stomatogastric ganglion (STG) of the crab, Cancer borealis [1-2]. In particular, the STG that contains the pyloric circuit in the crab stomatogastric nervous system, one of the two central pattern generators in the STG that are responsible for controlling the musculature of the digestive system. The pyloric rhythm (PR) controls the pylorus, which performs the mixing and filtering of food particles after chewing by the gastric mill inside of the crab stomach. Analytically, it is a classic model for motor pattern generation, where regular and predictable oscillatory activities of the participating neurons can be monitored and studied in vitro. In particular, a typical approach identifies neuron types participating in the PR by comparing the intracellular membrane potential oscillations and firing phases using extracellular captured activity patterns through the lateral ventricular nerve (lvn) [2-4].

This paper demonstrates the identification of pyloric neurons in the STG using the image/data obtained from the optical recordings with the VSDi approach described above without resorting to the difficult electrophysiological method of intracellular electrode recordings. Instead, the neural membrane potential oscillations in the STG 
were measured through the optically recorded intensity of the fluorescence produced by the voltage-sensitive dye (Di-4-ANEPPS) that was bath-applied to the STG exogenously. Several key challenges had to be overcome. (1) The relatively low yield ${ }^{1}$ of the potential-dependent fluorescence change $(2-10 \% / 100 \mathrm{mV})$ achieved by the Di-4ANEPPS dye used in our study make the PR extraction from the recorded dye bath/images a complex signal processing task [5]. (2) The inherently tri-phasic PR monitored on the lvn by the activity of three distinct types of neurons; namely, lateral pyloric (LP, one cell) neuron, the pyloric constrictor (PY, 5 cells) neurons and the pyloric dilator (PD, two cells) neurons, is recorded as extracellular neuronal potentials (i.e. spikes) corresponding to the highly coordinated oscillatory activities of the participating neurons. As with most extracellular electrode recordings of neuronal activity, such recording is typically displayed in the form of high-frequency sequence or train of spikes (i.e. bursts). Consequently, robust signal processing methods are needed to identify the network activities at a single-cell level, to facilitate analysing these spike trains (e.g. burst and network burst detection) in order to decode the (three) characterising phases of the PR. (3) The timing lag between the optical and lvn recordings of the characteristic spike peaks for the participating neurons due to the axonal transmission delay between the ganglion and the extracellular recording site is unknown and dependent upon (a) the biological/STG sample, (b) the equipment ensemble and (c) the experiment setup.

Our work required the development of a computationally robust solution that would reliably detect and extract the PR from the simultaneous measurement of membrane potential changes in the pattern generating neurons described in (1) above. This problem is discussed in [5]. In this paper, we present our work toward addressing the outstanding challenges.

\section{Duty Cycle of Component Neurons as Biometrics}

In electrical engineering, it is commonly known that duty cycles (DCs) can be used to specify the percentage time of an active signal in an electrical device such as the power switch in a switching/mode power supply or, relevantly, the firing of action potentials by a living system such as a neuron ${ }^{2}$. In the biological context [6-7], the relative phasing and DCs of the component neurons in a network are critically important for the generation of a specific behavior. Importantly, the ability of the pacemaker ensemble to maintain constant DC at different frequencies in the pyloric circuit of the STG could explain how the full network maintains fixed phase relationships at different frequencies [6].To maintain fixed phase relationships, neurons must all begin to fire later in the cycle as the cycle period increases. As the pacemaker ensemble releases transmitters in a voltage-dependent fashion during the burst when the duration of the pacemaker burst increases, the time during which inhibitory transmitter is released is accordingly extended. This, in turn, retards the onset of a firing of the

\footnotetext{
${ }^{1}$ https://www.thermofisher.com/order/catalog/product/D1199 last accessed 2/4/2017

2 Broadly, the DC of a neuron is defined as the ratio of the duration of the oscillator burst to the cycle period.
} 
follower neurons inhibited by the pacemaker network. Thus, the maintenance of constant phase in these follower neurons critically relies on the ability of the pacemaker ensemble to maintain a constant DC as frequency is changed. Recent studies on how pyloric neurons maintain their phase relationships with a relatively constant DC against temperature changes add further weight to such analysis [8].

\subsection{Ratio of Harmonics (RH)}

Computationally, the DC of a pyloric neuron can be defined as the percentage of the time, in each (pyloric) cycle, that the displayed waveform by the neuron is above its mean membrane potential. This enables studying of the waveform using the classical theory of Fourier/harmonics analysis. Specifically, any non-sinewave periodic waveform contains energy at harmonics of the fundamental frequency (f). This can be illustrated using a simple rectangular pulse wave/train as shown in Fig. 1 below, where the DC $(d=k / T)$ is defined as the fraction of time when the amplitude (A) of the wave is positive. Here, the Fourier series coefficients $\left(a_{n}\right.$ and $\left.b_{n}\right)$ are computed by correlating the waveform, $x(t)$, with cosine and sine waves over a full period $\mathrm{T}$ ( $1 / T$ ). The results, described in most textbooks (e.g. [9]), are given in equation (1) which provides a complete description (based on the Fourier series expansion) of the harmonics $(n f, n=1,2, \ldots)$ contained in $x(t)$. The latter can be used to characterize the pulse train and, in general, any periodic waveform ${ }^{3}$. In particular, the ratio of harmonics (RH), which has been used in recent years to measure symmetry, or relevantly, rhythmicity, as a defining characteristic in the investigation of the impact of various pathologies on locomotion [10], offers an amplitude independent measure of the periodic waveform of interest in this study; namely, $\mathrm{RH}_{21}=a_{2} / a_{1}$ and $\mathrm{R}_{31}=a_{3} / a_{1}$, which are calculated as $\frac{\sin 2 \pi d}{2 \sin \pi d}$ and $\frac{\sin 3 \pi d}{3 \sin \pi d}$ respectively.

$$
x(t)=A d+\sum_{n=1}^{\infty} a_{n} \cos 2 n \pi f t, \text { where } a_{n}=\frac{2 A}{n \pi} \sin n \pi d
$$

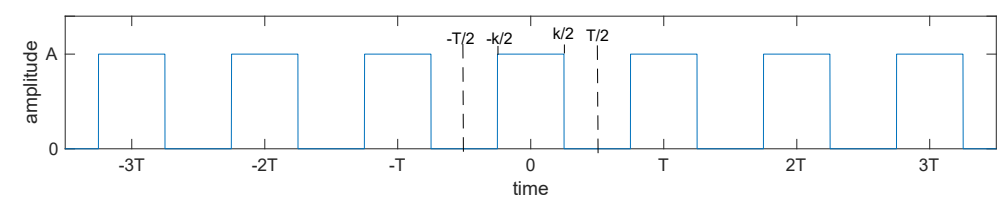

Fig. 1. An example pulse train $x(t)$ (blue) with a duty cycle (DC) calculated as the ratio of $k / T$.

\section{Experimental Setup}

Adult Cancer pagurus were obtained from Hodgkinson Fresh Fish, Manchester; they were kept in artificial seawater tanks (red sea salt, Red Sea) at $12-14^{\circ} \mathrm{C}$ with $12 \mathrm{~h}$

\footnotetext{
${ }^{3} \mathrm{NB}$. The $b_{n}$ coefficients in this example are all zeros, as $x(t)$ is symmetrical at $\mathrm{t}=0$ without loss of generality.
} 
light-dark cycle. The STG sample was prepared following the protocol described in [11] and was stained with Di-4-ANEPPS dye (Cambridge Bioscience, UK) and subsequently washed out using a saline flow.

The extracellular recording was executed on the lvn, with the resulting signal filtered and amplified by an AC differential amplifier before it was converted using Spike2 v8.07 (Power 1401, Cambridge Electronic Design, UK). The dyed STG was recorded using the MiCAM02 imaging system (SciMedia Ltd., Japan) simultaneously with the lvn recording. The optical data was extracted and exported using the BVAna imaging software (SciMedia Ltd., Japan) as a 3-D image stack written in CSV formatted spreadsheets, alongside a high-resolution image (HRI) of the sample. The HRI was used to identify neurons and select the pixels corresponding to the neurons as distinct regions of interest (ROIs). Signals corresponding to these ROIs were extracted from the 3-D stack in the form of 1-D time series data [5].

\section{$4 \quad$ Signal Processing}

To identify the pyloric neurons (PD, LP and PY) in the imaging data recorded from the dye-bathed STG, first we determined the three phases of the tri-phasic pyloric rhythm recorded on the lvn. This provided the discerning references with which the detected pyloric rhythm from the individual ROIs can be compared and analysed [5]. Here, so-called spike sorting techniques which were developed primarily to compare the waveforms of the individual spikes recorded extracellularly as "action potentials" of different neurons could be used [12]. However, while a detailed discussion or review on this approach is outside the scope of this paper, it should be noted that it is most difficult to identify the best algorithm with sufficient generality and also to define which spike sorter is the most appropriate given the task at hand [13]. In particular, the features-based approach that is primarily based on PCA and ICA to group spikes into clusters both rely on the orthogonality of components in a mixture and are unlikely to perform well, as the tri-phasic pyloric waveforms generated by the participating neurons are necessarily non-orthogonal [5]. Likewise, the template-based approach is unlikely to work well, as both the spatial and temporal variations displayed in the lvn would require the availability of an impractically large number of samples to be compared with the demonstrably noisy data obtained using the VSDi [5].

Our solution is formulated using the widely studied multi-resolution technique of time-frequency analysis to deconstruct the regular and predictable phasing of the pyloric activities recorded in the lvn [14]. Importantly, this is aided by the sequential Singular Spectrum Analysis (s-SSA) procedure, which we developed previously in [5] to extract the pyloric rhythm from the dye-bathed STG samples. As with the dyebathed image/data, the SSA-based procedure removed much of the noise from the lvn whilst preserving selectively the pyloric frequency and, relevantly, its higher order harmonics. The effect on the resulting spectrogram is depicted the Fig. 2. 

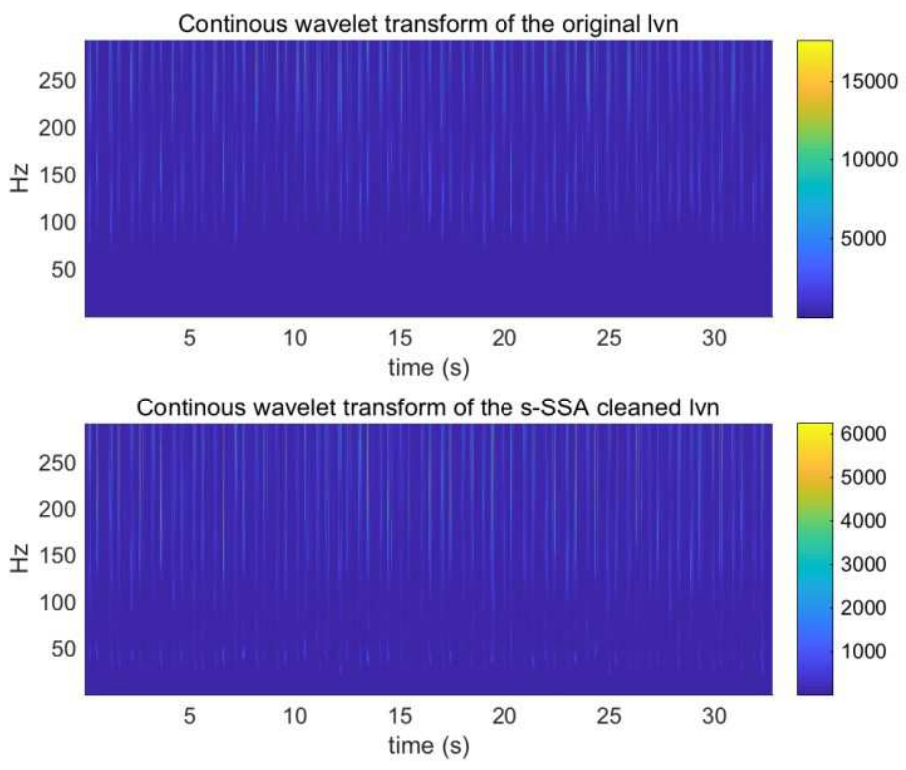

Fig. 2. Spectrograms generated with wavelet (Morse) based time-frequency decomposition. The improvement on clarity afforded to the frequency responses of the SSA processed lvn, particularly at the lower frequency bands, can be seen in bottom spectrogram. The colour bar accompanying each figure represents the amplitude computed by the continuous wavelet transform (CWT).

The analytic Morlet wavelet, known for its superior time localization, was used to better localize the transients between individual phases of the pyloric rhythm from the relatively noise free recording on the lvn. This was achieved by selecting in the constructed spectrogram a frequency band, where the amplitude response produced in the PY-led pyloric cycle is significantly lower than that in either of the LP-led and PD-led pyloric cycle. This is illustrated in Fig. 3, where the noise-free version of the lvn recording and the end-of-cycle marker $(*)$ are overlaid on the responses obtained in the selected frequency bands on which the PY-led cycles are effectively 'silenced'.

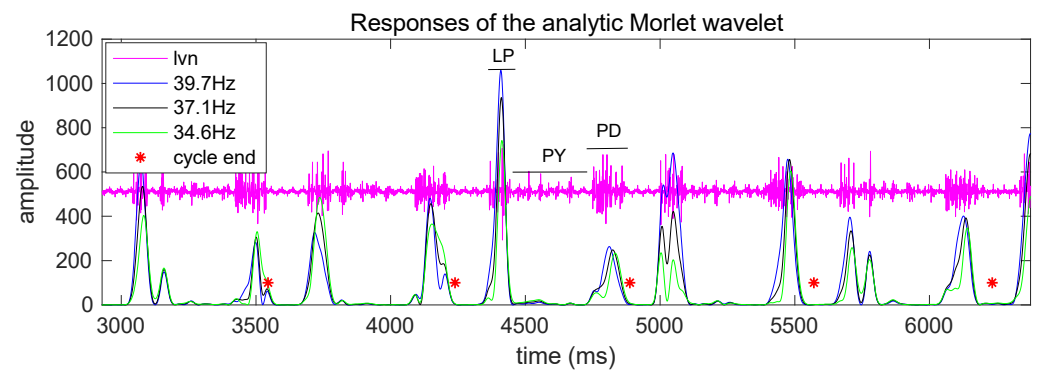

Fig. 3. Responses of the analytic wavelet in different frequency bands where the PY cycle is 'silenced'. The noise-free lvn is overlaid to demonstrate the accurate time localization of the transients between $\mathrm{LP} \rightarrow \mathrm{PY}$ and PY $\rightarrow \mathrm{PD}$ cycles. 


\section{$5 \quad$ Results and Discussions}

The transients of the PY bursts in each cycle obtained were used to reconstruct the PY phase of the pyloric cycle recorded in the lvn. The total wave energy of each cycle is also computed to approximate the average amplitude for the reconstructed pulse train; see Section 2.1. An example of the latter is shown in Fig. 4, where the PY phase/cycles were overlaid on the top of the lvn recording. Additionally, the duty cycle (DC) of each of these PY-led cycle/bursts were computed and their descriptive statistics calculated over the entire recording (32 pyloric cycles) are noted as follows: $\mathrm{DC}_{\text {mean }}=40.09 \%, \mathrm{DC}_{\text {s.t.d. }}=6.61 \%, \mathrm{DC}_{\min }=26.02$ and $\mathrm{DC}_{\max }=50.15 \%$.

The spectral content of the reconstructed PY burst train (depicted in Fig. 4) was examined by computing the periodogram as shown in Fig. 5d, where the pyloric frequency and its higher order harmonics are also noted; i.e., $\mathrm{PR}=1.017 \mathrm{~Hz}, \mathrm{RH}_{21}=$ 0.061 and $\mathrm{RH}_{31}=0.029$ which correspond to a DC of $41.5 \%, 38.75 \%$ and average DC of $\mathbf{4 0 . 1 3 \%}$ respectively. These parameters would be used, in the final validation step (described below), to compare with those obtained from the optically recorded dyebathed data in the ground truth set. The results are summarised in Fig. 5a, b \& c.

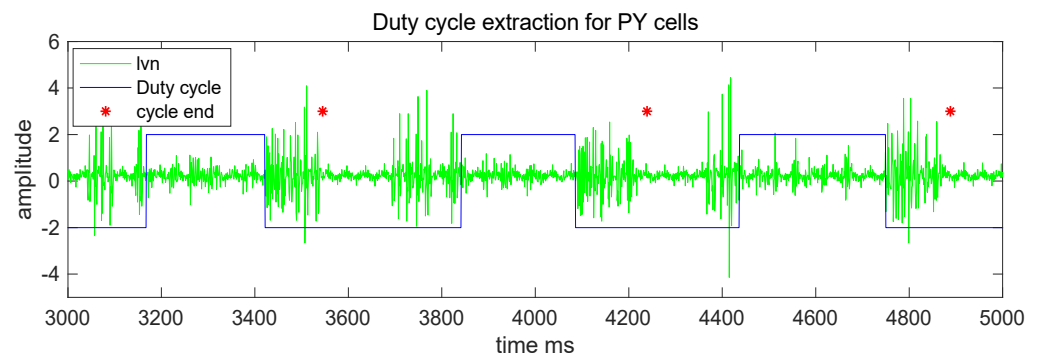

Fig. 4. Reconstruction of the PY burst train on the lvn recording (green background). The red asterisks are included to mark the end of each of the $\mathrm{PD} /$ burst cycle, to aid visual examination.

The pyloric cycle of the three (known) PY cells taken from the dye bath was extracted using the previously described s-SSA procedure in [5]. Here, it should be noted that this s-SSA procedure was adjusted to enable extraction of the higher order harmonic components of the pyloric frequency from the individual PY neurons. A periodogram is then constructed for each of these (PY) neurons in order to measure respective harmonics and, relevantly, the harmonic ratios, $\mathrm{RH}_{12}$ and $\mathrm{RH}_{13}$ from which the respective DCs can be calculated as follows: $\mathrm{DC}_{\text {cell_ } 1}=\mathbf{3 9 . 5 \%}, \mathrm{DC}_{\text {cell_ } 2}=\mathbf{3 9 . 9 \%}$, $\mathrm{DC}_{\text {cell } 3}=\mathbf{3 8 . 1} \%$. These figures represent the averaged $\mathrm{DC}$ values corresponding to the $\mathrm{RH}_{12}$ and $\mathrm{RH}_{13}$ shown in each figure (a, b and c) for the respective cells $(1,2$ and 3 ) in Fig. 5. Finally, the time delay between the optical and lvn recordings was computed using the Hilbert transform approach, whereby the instantaneous phase of the PY-cycle separate from each recording was obtained from the respective Hilbert spectra [15]. Here, the ability to effectively unpick the mono-frequency component of the 
pyloric rhythm by the s-SSA procedure developed in [5] was evident. The results are summarized in Fig. 6, where it is shown that the timing lag between the individual PY cell and the lvn maintains an expectedly constant mean phase shift of 3.34 radians (s.t.d $=0.24$ rads).
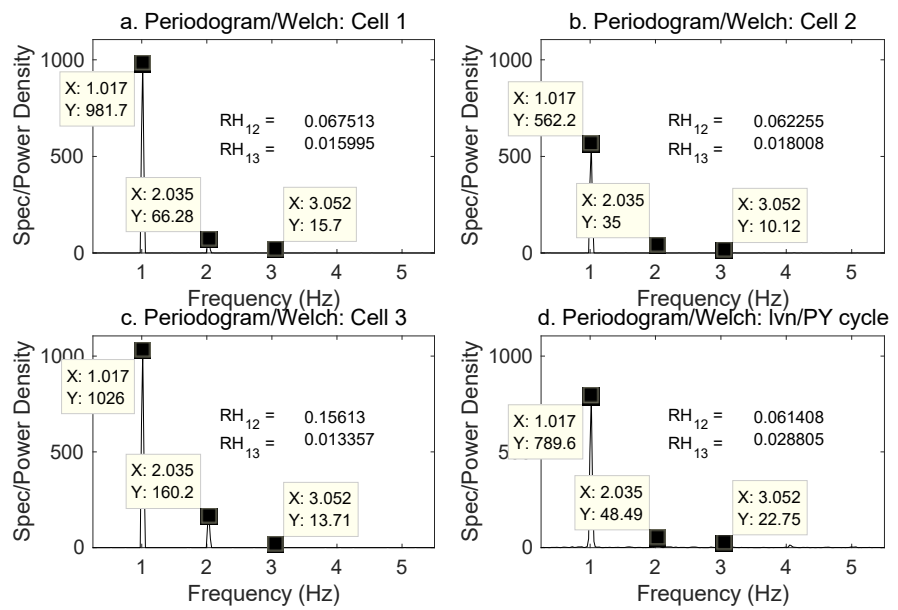

Fig. 5 Periodograms constructed for the individual cells (Fig. 5a,b\&c) and the reconstructed PY burst (Fig. 5d), showing the pyloric frequency, $2^{\text {nd }}$ and $3^{\text {rd }}$ harmonics at $1.107 \mathrm{~Hz}, 2.035 \mathrm{~Hz}$ and $3.052 \mathrm{~Hz}$ respectively. The ratios of harmonics, $\mathrm{RH}_{12}$ and $\mathrm{RH}_{13}$ are also given in each case.

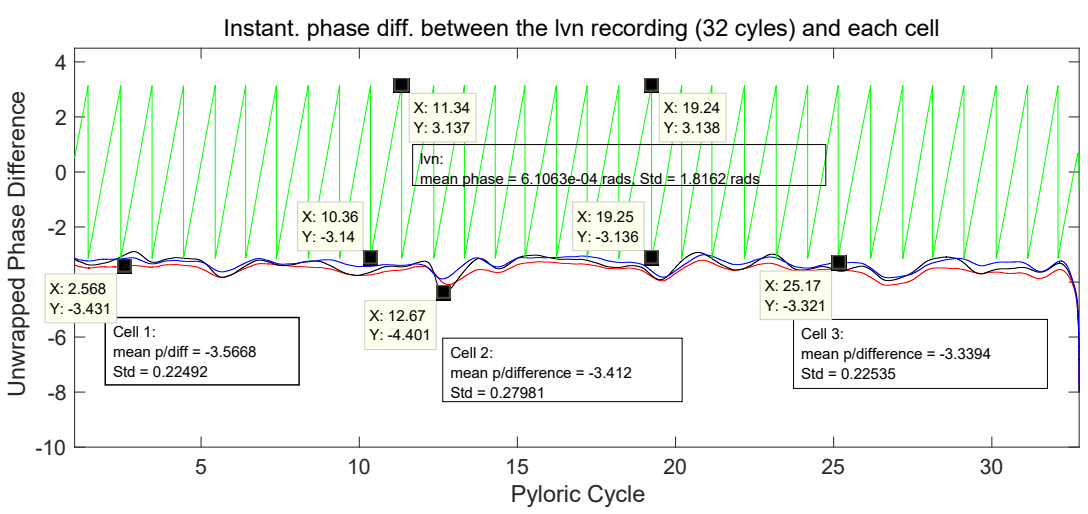

Fig. 6. Plots of the instantaneous phase shifts between the optical and lvn recordings obtained for the PY cells. The instantaneous phase changes of the lvn recording over the 32 pyloric cycles are also shown as a reference (green).

\section{Conclusions}

The ability to locate circuit neurons and simultaneously record from them at singlecell resolution is fundamental to understanding the full-scale network behaviour of neural systems. In STG, the traditional method to identify pyloric neurons by intra- 
cellular recording can be challenging and VSDi, an optical recording technique which simultaneously measures the membrane potential of multiple neurons, offers a promising solution. However, analysing features of VSDi based recordings of these neurons in relation to the PR obtained on the lvn is an exacting task, requiring the development of sophisticated computational/signal processing techniques that aim to decode the intrinsically tri-phasic pattern of the PR. Using DC as a biologically meaningful biometric, this paper demonstrates a practical means of achieving this aim. Importantly, the proposed solution required few manual adjustments and thus lends itself to potential real-time implementations.

\section{References}

1. Stein, W., Andras, P.: Light-induced effects of a fluorescent voltage-sensitive dye on neuronal activity in the crab stomatogastric ganglion. J Neuro. Methods, 188:290-294 (2010).

2. Stein, W., Städele, C., Andras, P.: Single-sweep voltage sensitive dye imaging of interacting identified neurons. J. Neuro Methods, 194:224-234 (2011)

3. Städele, C., Andras, P., Stein, W.:, Simultaneous measurement of membrane potential changes in multiple pattern generating neurons using voltage sensitive dye imaging. J. Neuroscience Methods, 203: 78-88 (2012)

4. dos Santos, F., Steyn, J.S., Andras, P.: Modelling the restoration of activity in a biological neural network, Int. Joint Conference on Neural Networks (IJCNN)(2016).

5. dos Santos, F., Andras, P., Lam, K.P.: A Multi-resolution Approach to the Extraction of the Pyloric Rhythm, to appear IEEE TSP, July, Barcelona, 2017.

6. Harris-Warrick, R.M. et al.: Dynamic Biological Networks, MIT Press (1992).

7. Golowasch, J., Manor, Y., Nadim, F.: Recognition of Slow Processes in Rhythmic Networks. Trends in Neuroscience, 22(9): 375-377(1999).

8. Tang, L.S. et al.: Precise Temperature Compensation of Phase in a Rhythmic Motor Pattern, PLoS Biology; 8/8 Aug (2010).

9. James H. McClellan: DSP First: A Multimedia Approach. Prentice Hall (1998).

10. Bellanca, J., Lowry K., VanSwearingen J., et al.: Harmonic ratios: a quantification of step to step symmetry, J Biomechanics 46 (2013).

11. Gutierrez, G., Grashow R.G.: Cancer borealis Stomatogastric Nervous System dissection, J. Vis. Exp., pp. 1-5, (2009).

12. Lewicki, M.: A review of methods for spike sorting: the detection and classification of neural action potentials. Network Comput. Neural Syst. 9, R53-R78 (1998).

13. Gibson S., Judy J.W., Markovic D.: Technology-aware algorithm design for neural spike detection, feature extraction, and dimensionality reduction, IEEE Transactions on Neural Systems and Rehabilitation Engineering, vol. 18, no. 5, pp. 469-478 (2010).

14. Akansu, A.N., Haddad R.A.: Multiresolution Signal Decomposition ( $2^{\text {nd }}$ Ed) Transforms, Subbands, and Wavelets, AP, 2000.

15. Hahn, S.: Hilbert transforms in signal processing, Artech House, Inc., Norwood, 1996. 